\title{
Erratum to: Heat transfer during drop impact on a heated foil
}

\author{
Tatiana Ponomarenko ${ }^{1,2,3}$ and Aleksey Safonov ${ }^{1,3}$ \\ ${ }^{1}$ Kutateladze Institute of Thermophysics SB RAS, 630090, Novosibirsk, Russia \\ ${ }^{2}$ Novosibirsk State Technical University, 630073, Novosibirsk, Russia \\ ${ }^{3}$ Novosibirsk State University, 630090, Novosibirsk, Russia
}

Original article: EPJ Web of Conferences 196, 00044 (2019), https://doi.org/10.1051/epjconf/201919600044

\section{The following affiliations:}

Tatiana Ponomarenko ${ }^{1,2}$ and Aleksey Safonov ${ }^{1,3}$

${ }^{1}$ Kutateladze Institute of Thermophysics SB RAS, 630090, Novosibirsk, Russia

${ }^{2}$ Novosibirsk State Technical University, 630073, Novosibirsk, Russia

${ }^{3}$ Novosibirsk State University, 630090, Novosibirsk, Russia

\section{Should be replaced by:}

Tatiana Ponomarenko ${ }^{1,2,3}$ and Aleksey Safonov ${ }^{1,3}$

${ }^{1}$ Kutateladze Institute of Thermophysics SB RAS, 630090, Novosibirsk, Russia

${ }^{2}$ Novosibirsk State Technical University, 630073, Novosibirsk, Russia

${ }^{3}$ Novosibirsk State University, 630090, Novosibirsk, Russia

\section{2. and Conclusion}

The first test experiments with a pulsating heat pipe with water were carried out. It is established that pulsations begin when the tube is heated. In the future, it is planned to use sapphire tubes to determine the water temperature

\section{The conclusion of the article should be replaced by the following text:}

The experiments with a drop impact on the thin heated constantan foil with fluoropolymer coating were carried out. It is demonstrated that diameter of the drop after impact is increasing due to dynamics of the drop and than decreasing due to evaporation effect. 